\title{
Signal characterization and assessment of code GNSS positioning with low-power consumption smartphones
}

\author{
Jacek Paziewski ${ }^{1}$ (i) $\cdot$ Rafal Sieradzki $^{1} \cdot$ Radoslaw Baryla $^{1}$ \\ Received: 13 November 2018 / Accepted: 9 July 2019 / Published online: 15 July 2019 \\ (c) The Author(s) 2019
}

\begin{abstract}
It has been acknowledged that smartphone GNSS observations suffer not only from high measurement noise and multipath but also from anomalies such as duty cycling and gradual accumulation of phase errors. These phenomena importantly constrain the application of smartphone phase measurement to high-precision techniques such as RTK or PPP. Hence, we aim at a comprehensive characterization of smartphone signal quality, including carrier-to-noise density ratio, measurement noise and anomalies present in observables with the focus on the impact of duty-cycling mode. The analysis confirms the abnormal properties of smartphone measurements related to the divergence between code and phase data and poor quality of the latter. To address these limitations, the second objective is to assess the smartphone medium- to long-range code-based relative positioning. This task includes the validation of the weighting scheme suited for handling the low quality of smartphone observations. The results show that it is feasible to use a sparse countrywide GNSS network as reference stations for codebased relative positioning. Even with the baselines over $100 \mathrm{~km}$, we can significantly enhance the positioning with respect to a stand-alone solution and reach the submeter level of horizontal coordinate accuracy. We have also noticed a discernible benefit from the C/NO-dependent weighting scheme, which is superior to the satellite elevation one.
\end{abstract}

Keywords GNSS pseudorange positioning · Smartphone positioning · Observation quality assessment $\cdot$ Smartphone GNSS observations

\section{Introduction}

Nowadays, we observe a rapid development in the low-cost GNSS chipsets, which are typically capable of tracking single-frequency multi-constellation signals. These include both low-cost but high-precision chipsets, e.g., produced by u-blox and SkyTraQ, whose application and high performance were proven in Takasu and Yasuda (2008), Wisniewski et al. (2013), and Odolinski and Teunissen (2017) and those of lower performance provided by, e.g., Broadcom, Qualcomm or Intel, which are commonly used in smart devices. The latter group of GNSS chipsets may be successfully applied to selected position-based services such as vehicle tracking, social networking and personal navigation, where typical accuracy of $2-3 \mathrm{~m}$ is sufficient (Wang et al.

Jacek Paziewski

jacek.paziewski@uwm.edu.pl

1 University of Warmia and Mazury in Olsztyn, Olsztyn, Poland
2011; Saeedi et al. 2014; Engelbrecht et al. 2015; Specht et al. 2019).

A milestone on the way to the introduction of smartphones to location-based applications was making GNSS observations derived from the devices running the Android Nougat 7 operating system accessible to all developers. This step allowed the acquisition not only of code pseudoranges but also phase and Doppler observations, which made it possible to develop algorithms enhancing the accuracy of positioning with mass-market devices. Consequently, the smartphone measurements have also become a subject of intensive analyses aimed at the evaluation of their quality. The initial studies conducted by Pesyna et al. (2014) were related to the application of the smartphone GNSS antenna combined with a high-grade receiver. The assessment revealed low multipath suppression of the smartphones' antennas but proved that achieving a centimeter-level position accuracy is feasible. A study conducted by Siddakatte et al. (2017) confirmed the low quality of the smartphone antenna but indicated a possible enhancement with the application of an external one. The authors 
also highlighted a strong influence of the $\mathrm{C} / \mathrm{N} 0$ values on code noise which can range between 2 and $15 \mathrm{~m}$. KirkkoJaakkola et al. (2015) aimed at the implementation of RTK using low-cost receivers. The results obtained with Nokia Lumia 1520 also indicated a low quality of smartphone measurements, which was reflected in high noise in the order of tens of meters for pseudoranges and frequent outliers. This resulted in a meter-level accuracy of RTK positioning. Laurichesse et al. (2017) reported, however, a submeter accuracy of single point positioning supported with SBAS corrections and Doppler filtering.

Several contributions considered precise positioning with the Google/HTC Nexus 9 tablet, which is capable of collecting continuous phase observations, whereas the vast majority of smartphone GNSS chipsets takes advantage of hardware clock discontinuity to support power-save duty cycling. Realini et al. (2017) proved the feasibility of the Nexus 9 measurements for close-range relative positioning with a single baseline up to several kilometers. In such a case, it was likely to obtain centimeter-level accuracy. Gill et al. (2017) analyzed single-frequency precise point positioning (PPP) and achieved the accuracy of several decimeters. To follow on that, an important study was recently conducted by Zhang et al. (2018b). The authors proposed a time-differencing filtering approach for enhancement of positioning performance, which led to the horizontal and vertical position errors at the level of $0.8 \mathrm{~m}$ and $1.4 \mathrm{~m}$, respectively.

Other studies verified the applicability of the devices which suffer from duty cycling. The investigations given by Humphreys et al. (2016) confirmed the poor quality of phase data, which are affected by discontinuities and gradual accumulation of errors. The latter effect may be related to the fact that the smartphones commonly log accumulated delta ranges with an arbitrary phase offset instead of true fullphase observations (Riley et al. 2017). This prevents ambiguity fixing and forces application of float solution. Liu et al. (2018) reported improvement of the performance of closerange relative positioning taking advantage of pseudorange smoothing. The pedestrian navigation algorithm that considers the duty cycling was proposed in Liu et al. (2019). Nonetheless, the results are still not sufficiently conclusive.

We focus on the challenging case with an important constraint, which is enabled duty cycling to low-power consumption smartphones. The starting point of this work is aimed at the evaluation of smartphone observables with particular emphasis on anomalies present in phase and code observables driven by the duty-cycling mode. According to the results of this initial part and addressing the phase measurement limitations, the second objective is to assess the medium to long-range code-based relative positioning. We aim to evaluate the accuracy level that can be reached with such devices and to validate the weighting scheme suited for handling the low quality of smartphone observations.
In the following section, we discuss the carrier-to-noise density ratio, quality and noise of smartphone observables with the special focus on the impact of duty cycling. Then, we evaluate the positioning accuracy level with smartphonederived measurements and described potential benefits from $\mathrm{C} / \mathrm{N} 0-$ dependent weighting function in the pseudorange relative positioning of different ranges. Finally, conclusions are drawn in the last section.

\section{Smartphone observation quality assessment}

This section provides selected properties of the smartphone GNSS measurements. We investigate carrier-to-noise density ratio, anomalies in smartphone data as well as observation noise.

\section{Experiment design and data collection}

The observation quality assessment is based on signals collected by smartphone affected by duty cycling and highgrade receivers: Huawei P20, Javad Alpha + GrAnt_G3T antenna and Javad Triumph, respectively. The smartphone logged GPS + Galileo + BDS measurements with 1-s interval to RINEX 3.03 over a timespan of about $13 \mathrm{~h}$ (3:50-17:00 UTC) on May 11, 2018, with the use of Geo ++ RINEX Logger ver. 1.2.5. Three collocated receivers formed ultra-short baselines of several meters under an unobstructed sky environment. The smartphone was centered above a temporal geodetic site with the use of tripod and tribrach (Fig. 1). This allowed for the elimination of centering errors and further determination of benchmark coordinates of the site with high-grade receiver set. The processing was done with multi-purpose GNSS software (Paziewski 2016).

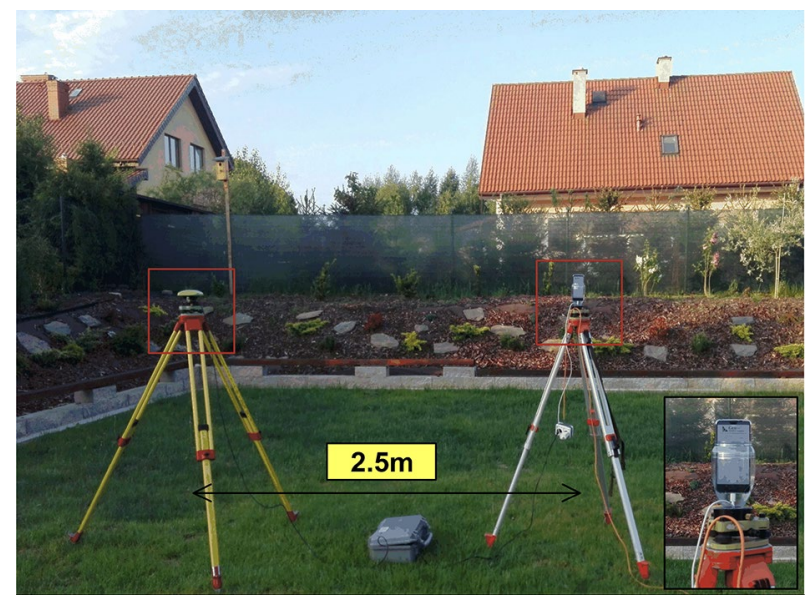

Fig. 1 Huawei P20 smartphone collocated with Javad Alpha+GrAnt_G3T during data collection 


\section{Satellite signal power analysis}

Carrier-to-noise density ratio $(\mathrm{C} / \mathrm{N} 0)$ is defined as the ratio of the signal carrier power to the noise power in a $1 \mathrm{~Hz}$ bandwidth. This indicator strongly depends not only on the satellite antenna, signal propagation and deterioration but also on the hardware, including antenna, receiver and cables (Wang et al. 2012; Hauschild et al. 2012).

Figure 2 depicts $\mathrm{C} / \mathrm{N} 0$ values collected by the collocated receivers: Javad Alpha and the smartphone. Just a brief look at the figure gives us the impression of a lower level of $\mathrm{C} /$ $\mathrm{N} 0$ of the signals collected by the smartphone in comparison with the high-grade receiver. In general, the carrier-to-noise density ratio of signals collected by Javad fits in the range of $35-55 \mathrm{~dB}-\mathrm{Hz}$, whereas in the case of the smartphone, these values did not exceed $48 \mathrm{~dB}-\mathrm{Hz}$.

Figure 3 provides the histogram of the $\mathrm{C} / \mathrm{N} 0$ differences of the corresponding signals collected by the employed receivers. The results reveal that the $\mathrm{C} / \mathrm{N} 0$ of smartphone measurements is about $9.4 \mathrm{~dB}-\mathrm{Hz}$ lower with respect to the Javad receiver. These results are in line with the conclusions drawn by Zhang et al. (2018a), Gill et al. (2017) and Liu et al. (2019) who showed that C/N0 of raw smartphone measurements are in general $7.5-10 \mathrm{~dB}-\mathrm{Hz}$ lower comparing to the high-grade hardware.

Figure 4 presents $\mathrm{C} / \mathrm{N} 0$ values with respect to the satellite elevation angle. As expected, the $\mathrm{C} / \mathrm{N} 0$ values logged by the high-grade receiver are characterized by a clear relationship
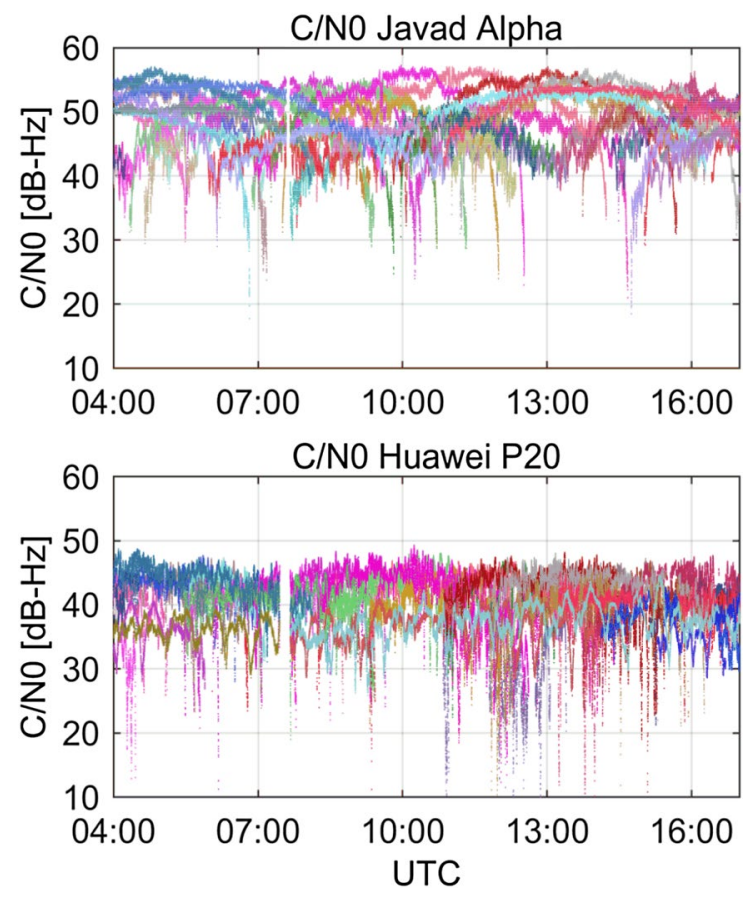

Fig. 2 Time series of C/N0 values collected by Javad Alpha (top) versus Huawei P20 (bottom)

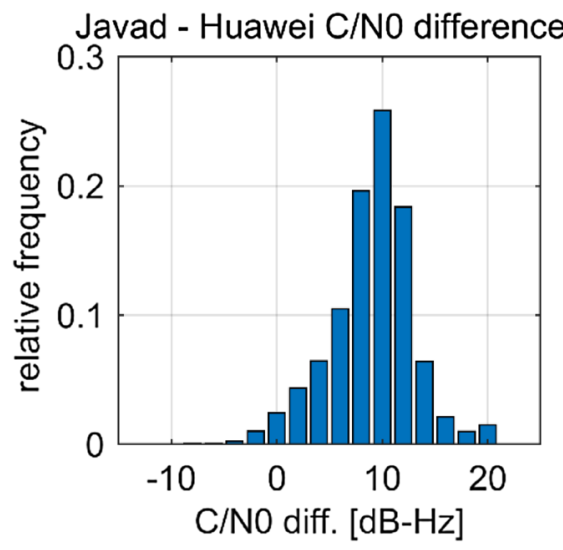

Fig. 3 Histogram of the C/N0 differences between corresponding signals collected by Javad Alpha and Huawei P20

with satellite elevation. However, we cannot draw similar conclusions in the case of the smartphone, since the plot is much more flattened. The relationship between the satellite elevation and $\mathrm{C} / \mathrm{N} 0$ of smartphone measurements is not obvious, especially at low elevations. In this case, the signals are characterized by a noticeable number of low values of $\mathrm{C} / \mathrm{NO}$, reaching the level of $10 \mathrm{~dB}-\mathrm{Hz}$, also noticed in Fig. 2. The occurrence of these outliers is probably related to multipath affecting the smartphone observables (Pesyna et al. 2014).
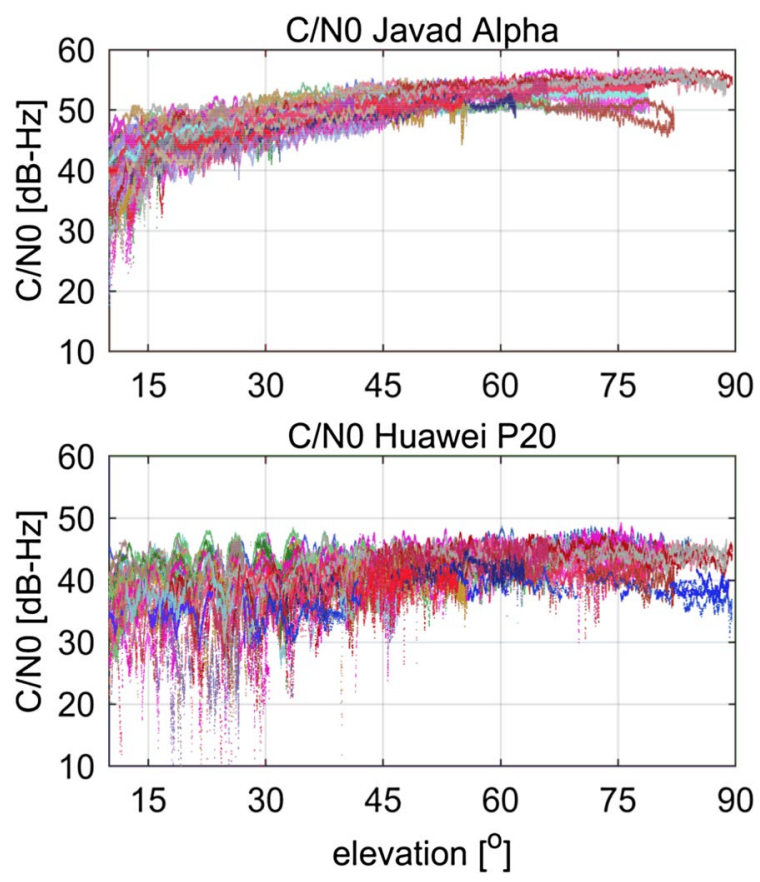

Fig. $4 \mathrm{C} / \mathrm{N} 0$ in the function of elevation angle of collected by Javad Alpha (top) versus Huawei P20 smartphone (bottom) 
Figure 5 gives us the impression of azimuthal-elevation dependence of $\mathrm{C} / \mathrm{NO}$ values. We notice a slight azimuthal asymmetry as well as the presence of patches of low signal power or even lack of signals for a mobile device, which are absent in the case of the high-grade receiver. This coincides with the investigations of Humphreys et al. (2016) who indicated a high impact of multipath affecting smartphone measurements. The results are given in Figs. 4 and 5 which suggest that the application of C/N0-dependent weighting scheme seems to be more appropriate than the elevation dependent. Hence, investigations in this field are conducted in the section devoted to positioning performance assessment.
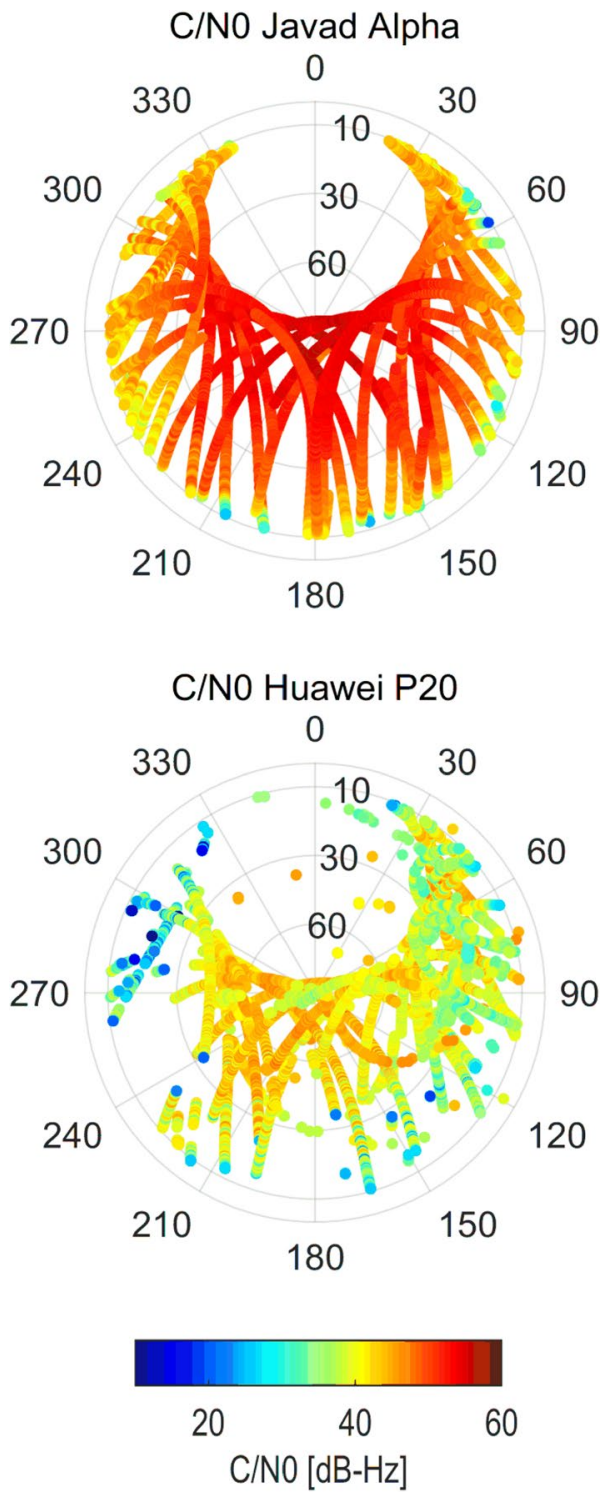

60

Fig. 5 Skyplots of C/NO collected by Javad Alpha (top) versus Huawei P20 (bottom)

\section{Characterization of smartphone observables with the focus on the impact of duty cycling}

We begin the smartphone observation analysis with the presentation of phase-code $(\mathrm{P}-\mathrm{C})$ differences. This combination is commonly used to assess the code noise; however, in this case, these values can provide us the first glance on the potential presence of effects related to the duty cycling. The phase-code combination between satellite $m$ and receiver $k$ in the units of meters can be expressed as follows:

$$
\begin{aligned}
\lambda \varphi_{k}^{m}-P_{k}^{m}= & \lambda N_{k}^{m}+c\left(B_{k, \varphi}-B_{\varphi}^{m}-B_{k, P}+B_{P}^{m}\right) \\
& -2 I_{k}^{m}+M_{k, \varphi}^{m}-M_{k, P}^{m}+\varepsilon_{\varphi}-\varepsilon_{P}
\end{aligned}
$$

where $\lambda$ is the signal wavelength, $\varphi$ is the carrier phase observable in cycles, $P$ denotes the code pseudorange, $N$ states for integer ambiguity, superscript $m$ and subscript $k$ denote satellite and station, respectively, and $B$ corresponds to receiver/satellite bias. When a subscript is present, this bias refers to a receiver, otherwise to a satellite. $I$ denotes ionospheric delay, $M$ is the multipath effect, and finally $\varepsilon$ denotes observation noise.

Figure 6 depicts the example time series of phase-code differences given for Huawei P20 versus Javad Alpha together with the signal $\mathrm{C} / \mathrm{N} 0$ during the first $20 \mathrm{~min}$ of data collection. For the clarity of presentation, we have
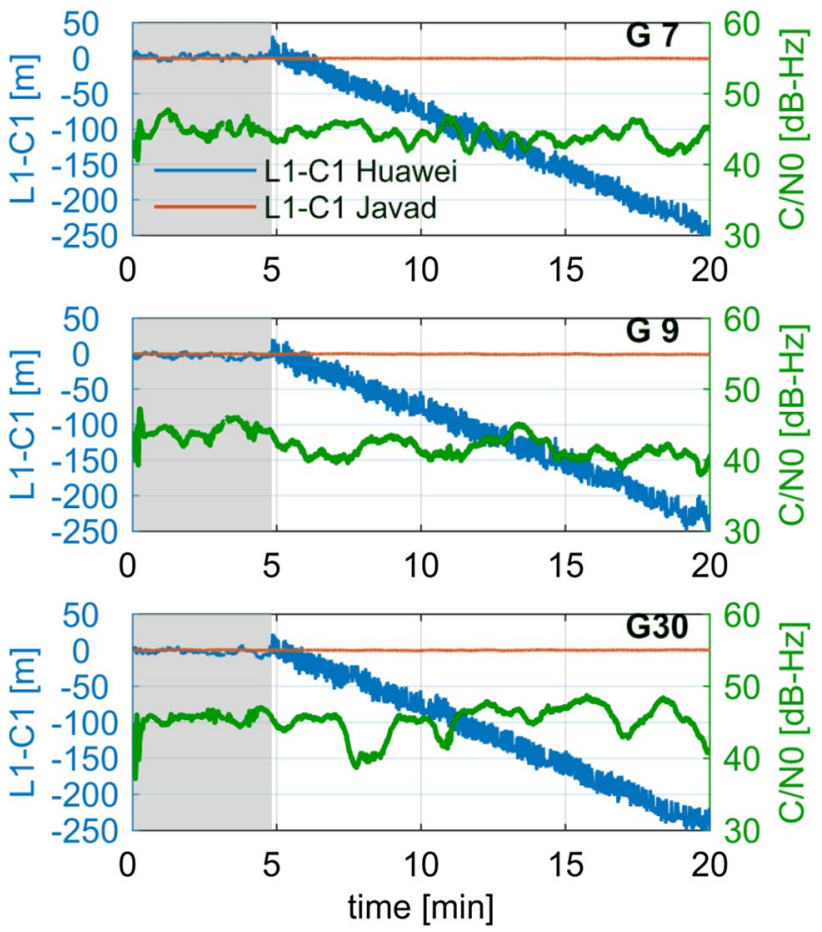

Fig. 6 Time series of phase-code differences (blue-Huawei, redJavad) and C/N0 of Huawei observables (green). The gray patch corresponds to the initial 5-min period with inactive duty-cycling mode 
corrected each time series with the value related to initial epoch. Thus, the obtained variations should correspond only to time-dependent parameters such as the ionospheric delay, multipath and observational noise without parameters considered as constant like ambiguities and hardware biases. This statement holds true for observables of the high-grade receiver since these phase-code differences are mainly driven by code measurement noise and a very weak trend related to the impact of the ionosphere. The similar behavior is noticed only during the initial 5-min-long period in the case of smartphone data (Figs. 6 and 7). This is related to the fact that duty cycling is activated by the smartphone after the time, which is required by the chipset for ephemerides acquisition (Pirazzi et al. 2017). It usually takes several minutes, which coincides with our results. We can find that after the initial period of approximately $5 \mathrm{~min}$, the smartphone phase-code differences become the subject of two anomalies. The first of them is a noticeable drift, responsible for increasing divergence between phase and code data. The latter is repeated variations featured with 3 -s periodicities and causing the jumps in phase-code data in the range of about $20 \mathrm{~m}$. Thus, we inclined toward the hypothesis that the smartphone observables anomalies are driven by dutycycling mode. The pattern of anomalies is observed for all satellites and due to the scale of variations should be rather considered as receiver clock-related effect (Fig. 6).

To verify the phenomena detected in $\mathrm{P}-\mathrm{C}$ differences, we have investigated phase and phase-code single differences (SD) using observations from collocated Javad and Huawei receivers. In this case, we consider tropospheric delays as greatly reduced due to the ultra-short distance between the receivers. Furthermore, we have eliminated the satellite-to-receiver distance and the receiver clock corrections derived from absolute code pseudorange solution with
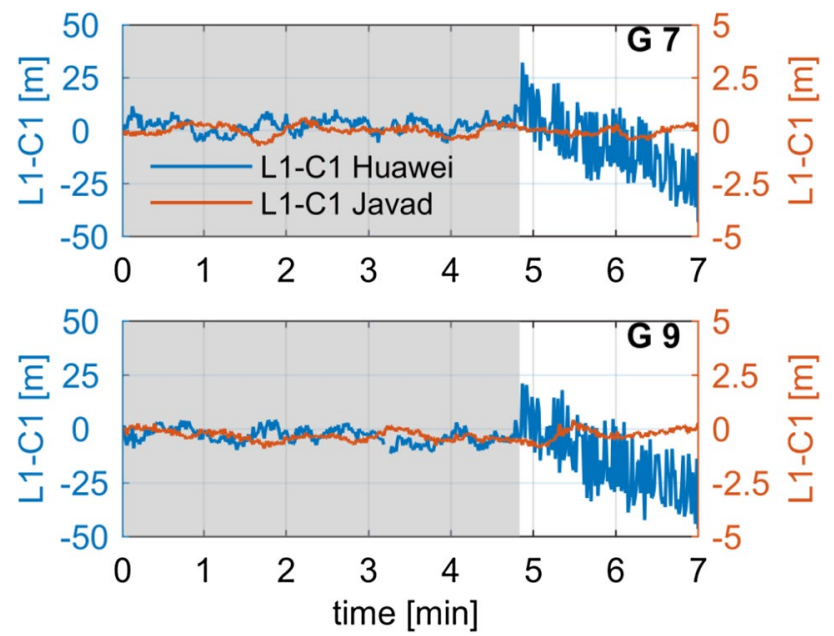

Fig. 7 Time series of phase-code differences for Huawei (blue) versus Javad (red) with the focus on the inactive duty-cycling period fixed coordinates. These smartphone clock corrections were smoothed by means of 30 epoch moving average to extract the information which observable is affected by the phenomena indicated in Fig. 6. One can expect that such SD phases should be the subject of ambiguities, observational noise, multipath, receiver biases and residual receiver clock term. The phase-code SDs are contaminated by the same factors as well as by doubled ionospheric delay. Considering the variations related to clock residuals as dominant, the between-receiver differences should allow for assignment of anomalies to particular observables.

Looking at Fig. 8, we can again notice unusual effects in both phase and phase-code SD after the activation of duty cycling. Phase SDs are the subject of strong drift observed earlier in smartphone phase-code combination (Fig. 6). Its occurrence leads to the conclusion that this effect is originated in the smartphone phase observables. Such gradual divergence confirms the inconsistency between smartphone phase and code clocks during the duty-cycling period. The effect needs further investigations, but it may be related to providing the accumulated delta range instead of true full phase. High variability of phase-code SD during the dutycycling period and lack of this effect in the SD phase indicate the smartphone code observables as a source of this phenomenon. The corresponding variability of the receiver clock correction estimated with code pseudoranges justified the origin of the effect. The occurrence of both anomalies
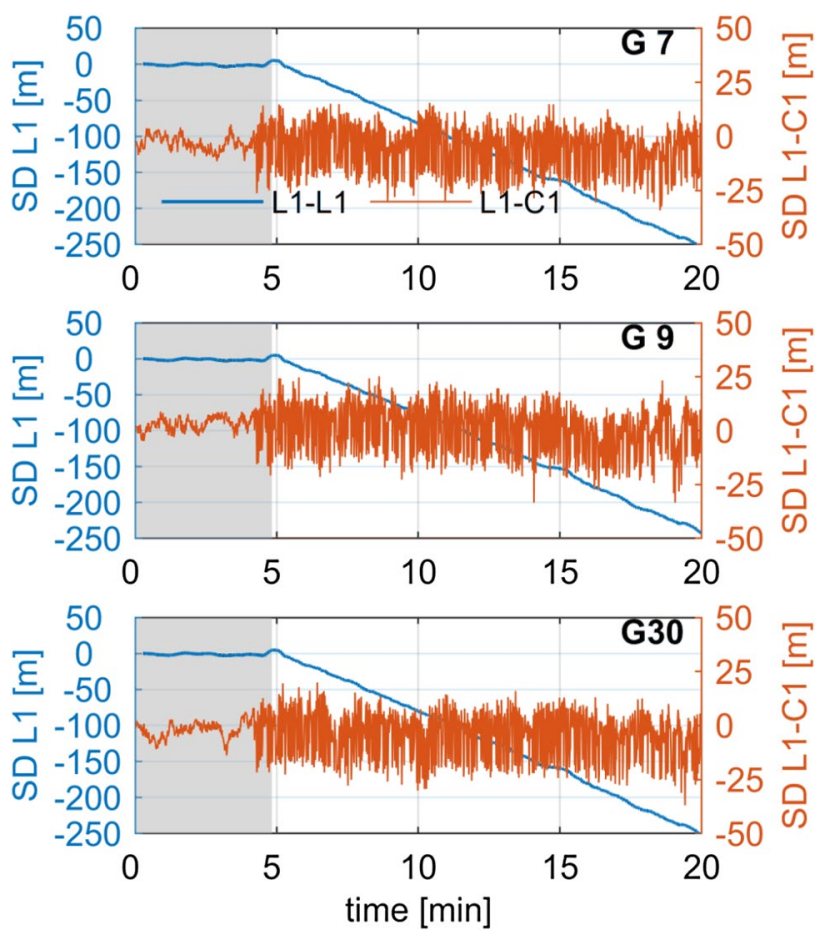

Fig. 8 Time series of between-receiver (Javad Alpha-Huawei P20) phase (blue) and phase-code (red) single difference 
suggests the necessity to estimate independently phase and code clock corrections in potential application of smartphones to PPP.

Figure 9 depicts the smartphone observables, which were doubly differenced in the time domain. Such combination greatly reduces the impact of geometric distance, receiver clock drift as well as satellite-dependent or spatially correlated errors. Thus, the amplified residuals illustrate only the impact of noise combined with remaining rapidly changing factors such as indicated above clock variations. From the figure, one can notice an increase in phase residuals after the activation of duty cycling reflected in the jump of standard deviations (STDs) from 0.10 up to $0.3-0.5$ cycle, depending on the satellite. During the initial 5-min-long period, the phase residuals of all satellites are highly correlated (about $0.9)$, which indicates the dominant impact of receiver clock residuals. During duty-cycling mode, the correlation coefficient is close zero, and thus, the increase in STD should be rather considered as an increment of phase measurement noise.

The similar pattern of time differences is observed for code observables. However, we believe that in this case, the increase in code residuals is mainly driven by receiver clock-related variations and, to a lesser extent, code noise. Dominating impact of the former factor is justified by the

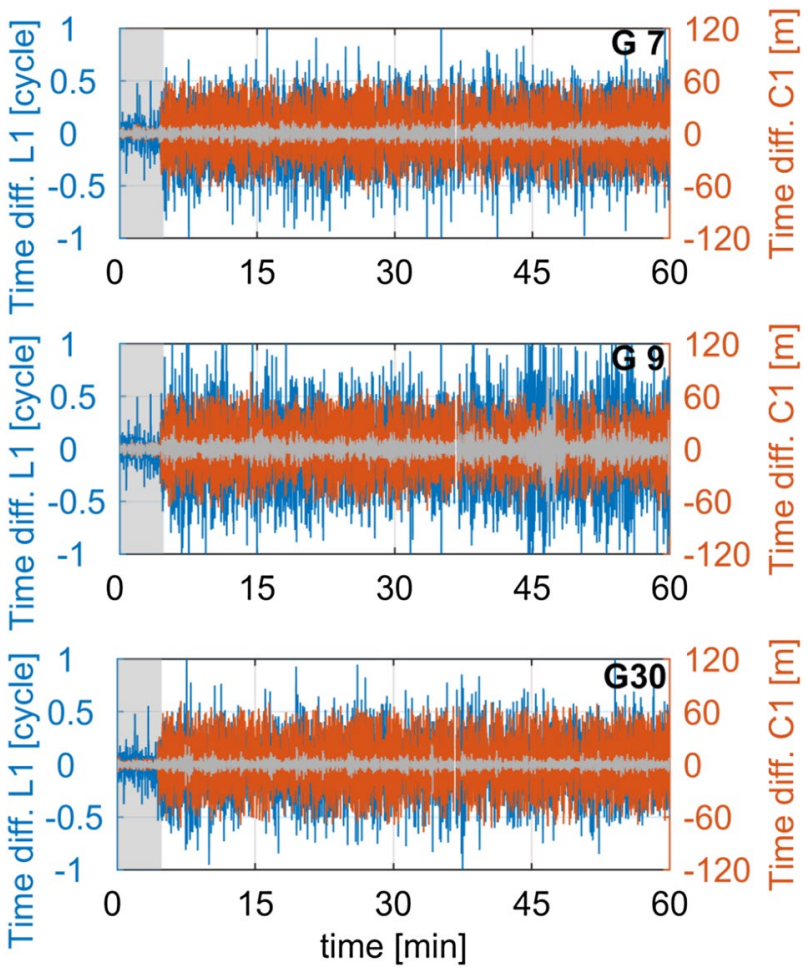

Fig. 9 Smartphone phase (blue) and code (red) double differences in the time domain. The code time differences with the preliminary elimination of receiver clock are given in the gray color high correlation between satellite time series during the duty-cycling period, which reached the level of 0.75 . Nevertheless, one can observe the noticeable growth of noise in code double differences with the preliminary elimination of receiver clock (Fig. 9).

Finally, we analyze double differences (DD) formed for grade receiver-smartphone ultra-short baseline. It is expected that in this case, we cancel all satellite- and receiver-dependent errors as well as highly mitigate the effects related to the propagation in the atmosphere. After the elimination of DD satellite-to-receiver distance, the phase time series should be only the subject of ambiguities as well as observational noise and multipath. Consequently, these time series should be free from any anomalies detected earlier, assuming that their pattern is the same for all satellites. For the clarity of presentation, we have removed the initial value from each arc of the DD phase residual time series.

Figure 10 shows that the previously detected strong trend and jumps have been eliminated in a great extent, but still we can see unwanted long-term drift in the phase data during the activated duty-cycling mode. In contrary to previously detected effects in SD, the phase DD does not reveal coincidence between different pairs of satellites and thus cannot be linked with the receiver clock. This is a surprising effect if we consider DD ambiguities as constant values, unlike in the case of high-grade receivers. At this step, it is hard to explain
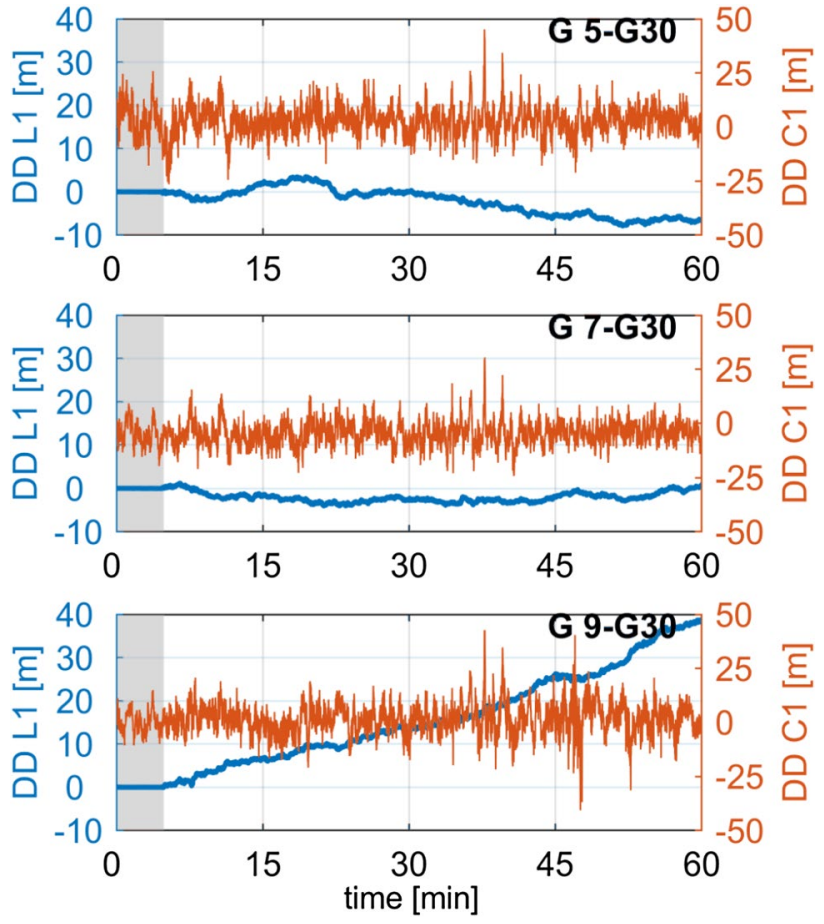

Fig. 10 Time series of double-differenced observations for ultrashort-baseline formed of high-grade receiver and smartphone 
this anomaly without the detailed knowledge of the smartphone signal acquisition algorithm. Nevertheless, the similar variations for DD phase data were previously reported by Humphreys et al. (2016) and Håkansson (2019). The authors justified their presence in zero-baseline DD phase observations by the gradual accumulation of the phase errors. The latter work also suggests a frequency deviation in phase tracking as a source of this anomaly. It should be remarked that these previous contributions analyzed duty-cycling free receivers and the drift depicted there was characterized by a smaller magnitude in relation to the results given in Fig. 10 . This behavior of phase observations seems to importantly constrain the application of smartphone phase observations to precise positioning. Hence, at this step, we consider the phase observations collected by smartphones with enabled duty cycling as inefficient for direct processing in relative or absolute mode.

On the other hand, looking at the code DD we cannot detect any anomalies after activation of the duty cycling, and hence, these were eliminated by double differencing. To address the above findings, in the next part of investigations, we focus on the application of code observations and determine the accuracy of positioning to be as high as possible in such a case.

\section{Observation noise}

GNSS signal noise characteristics may be appraised taking advantage of post-fit observation residuals or using time series of DD observations for zero baselines (Bona 2000; de Bakker et al. 2012). Since most of the smartphone devices are not equipped with external antenna connectors, such a case is not possible. Hence, we employed ultra-short baselines formed of Huawei P20 smartphone and two high-grade geodetic receivers with known benchmark coordinates. To retrieve measurement noise combined with the multipath effect, we took advantage of double- and triple-differenced (TD) observables with the eliminated impact of satelliteto-receiver geometry by means of a position-fixed model (Paziewski et al. 2018). Such DD time series for ultra-short baseline can be regarded as free from the influence of ionospheric and tropospheric delays, hardware biases, clock errors and geometry (Amiri-Simkooei and Tiberius 2007). Assuming the ambiguities as constant, the variations of DD should be predominantly related to the noise of signals and multipath effect. However, as shown in Fig. 10, this is not true for smartphone DD phase observables. Hence, considering the detected anomaly, we used also time differencing to reduce long-term drift and to isolate observable noise. We should note that due to the observables differencing the noise is amplified with respect to the undifferenced data, but the impact of multipath effect for 1-s TD time series is expected to be reduced to a great extent (de Bakker et al. 2012). Finally, DD and TD combinations for code and phase data can be written as follows:

$P_{k l}^{m n}-\rho_{k l}^{m n}=\varepsilon_{k l, P}^{m n}+M_{k l, P}^{m n}$

$\lambda \varphi_{k l}^{m n}-\rho_{k l}^{m n}=\lambda N_{k l}^{m n}+\varepsilon_{k l, \varphi}^{m n}+M_{k l, \varphi}^{m n}$

$P_{k l, t_{i j}}^{m n}-\rho_{k l, t_{i j}}^{m n}=\varepsilon_{k l, P, t_{i j}}^{m n}$

$\lambda \varphi_{k l, t_{i j}}^{m n}-\rho_{k l, t_{i j}}^{m n}=\varepsilon_{k l, \varphi, t_{i j}}^{m n}$

where the satellite $(m, n)$-to-receiver $(k, l)$ distance $(\rho)$ was computed using fixed a priori coordinates.

Table 1 demonstrates the statistics of double- and tripledifferenced residuals for the baselines formed of Huawei P20_Javad Alpha versus Javad Alpha—Javad Triumph receivers. The results show a clear drop in standard deviation after time differencing. Except for the smartphone phase data during the duty-cycling mode, in other cases, it is thought to be caused by the reduction in multipath, and effect was observed during the initial period in Fig. 7. High multipath is basically expected for smartphone observables, while its impact in the case of high-grade receivers is most likely due to the application of survey-grade antenna instead of chock ring. The outstanding STD for the DD phase during active duty cycling is a clear consequence of long-term drifts depicted in Fig. 10. The time differencing eliminated the unwanted phase drifts to a great extent. Nevertheless, looking at the TD result, one can assume that fixing of ambiguities during duty-cycling mode would not be possible even after the elimination of the anomaly.

It becomes clear, from the statistics given in Table 1, that phase and code observables are much noisier after activation of duty cycling, which is consistent with the results given in Fig. 9. In the case of code data, the activation of the duty cycling resulted in $24 \%$ and $56 \%$ increase in the standard deviation for DD and TD residuals, respectively. Analyzing the phase TD, one can observe over the sixfold increase in

Table 1 Standard deviation of double- and triple-differenced observable residuals $(\mathrm{m})$

\begin{tabular}{lllll}
\hline Observable & Combination & $\begin{array}{l}\text { Smartphone-high- } \\
\text { grade receiver baseline }\end{array}$ & $\begin{array}{l}\text { High-grade } \\
\text { receivers } \\
\text { baseline }\end{array}$ \\
\cline { 3 - 4 } & & $\begin{array}{l}\text { Inac- } \\
\text { tive duty } \\
\text { cycling }\end{array}$ & $\begin{array}{l}\text { Active } \\
\text { duty } \\
\text { cycling }\end{array}$ & \\
\hline Code & DD & 6.42 & 7.95 & 1.14 \\
& TD & 4.43 & 6.90 & 0.49 \\
Phase & DD & 0.0300 & 8.5120 & 0.0069 \\
& TD & 0.0145 & 0.0921 & 0.0024 \\
\hline
\end{tabular}



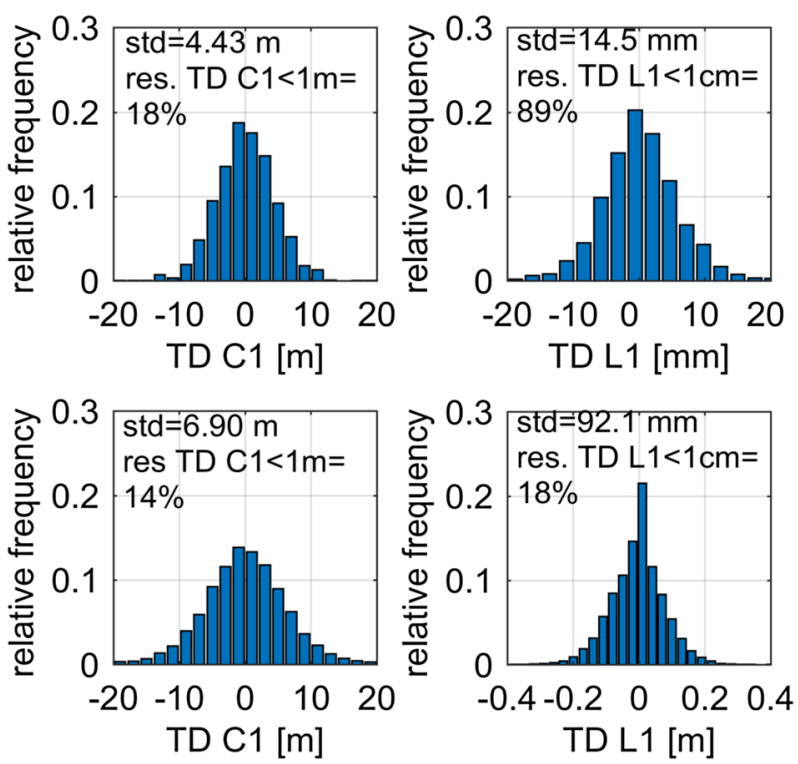

Fig. 11 Histogram of the TD observable residuals for Javad AlphaHuawei P20 baseline during inactive (top) and active (bottom) duty cycling
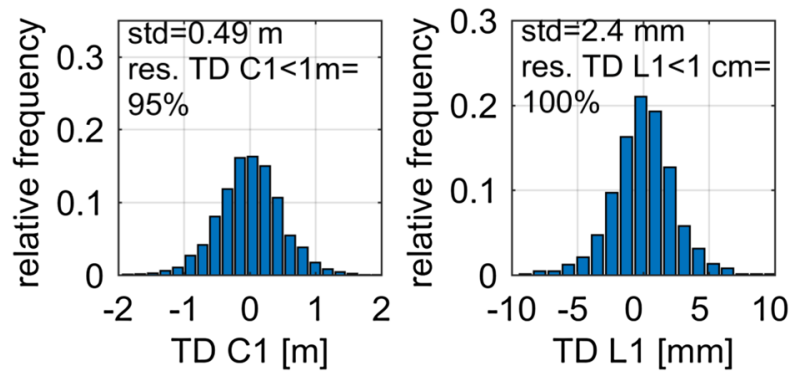

Fig. 12 Histogram of the TD observable residuals for Javad AlphaJavad Triumph baseline

STD up to $92 \mathrm{~mm}$ with respect to the period with inactive duty cycling.

As illustrated in Figs. 11 and 12, the TD code observations of the smartphone-high-grade receiver baseline are in general over ten times noisier than these of Javad receiver baseline. In the former case, we obtained the standard deviation of the TD code during inactive duty cycling at the level of $4.4 \mathrm{~m}$ with residuals fitting the range of $\pm 20 \mathrm{~m}$. In the latter case, STD reached only $0.5 \mathrm{~m}$ with dispersion in the range of $\pm 1.5 \mathrm{~m}$. In general, the ratio of TD code residuals fitting the range of $\pm 1 \mathrm{~m}$ reached the level of $95 \%$ in the case of the baseline of high-grade receivers, but only $18 \%$ in the case of the smartphone-high-grade geodetic receiver baseline during inactive duty cycling. As is the case for code pseudoranges, the TD phase observations for mixed receiver baseline are much noisier with respect to the baseline formed of high-grade receivers. In the former case, STD reached
$14.5 \mathrm{~mm}$ during inactive duty cycling. We can conclude that the smartphone observables are mainly responsible for high noise in this case. Regarding high-grade receivers, we obtained the expected level of $2.4 \mathrm{~mm}$. In this context, we consider the results obtained during inactive duty cycling as more reliable indicators of the smartphone observable noise, since after the activation of the algorithm, still, some anomalies may occur.

\section{Positioning performance assessment}

In this section, we present positioning performance assessment based on smartphone code observations. Taking into account the result of signal power and noise analyses, we investigate a potential benefit from relative positioning of different ranges based on the countrywide CORS network supported by $\mathrm{C} / \mathrm{N} 0$-dependent weighting scheme.

\section{Functional and stochastic modeling}

We employed a well-known geometry-based relative functional model limited to the application of code pseudoranges:

$P_{k l}^{m n}=\rho_{k l}^{m n}+I_{k l}^{m n}+T_{k l}^{m n}+M_{k l, P}^{m n}+\varepsilon_{k l, P}^{m n}$

Because only single-frequency signals are available, it is not feasible to apply advanced methods for ionospheric delay handling. Hence, ionospheric delay is reduced only by means of double differencing. However, in the case of smartphone relative positioning, we consider observation noise combined with the multipath effect as a dominating source of accuracy degradation rather than ionospheric delay. The tropospheric delay $(T)$ mitigation is supported by the modified Hopfield model with standard atmospheric parameters and GMF mapping function (Boehm et al. 2006; Wielgosz et al. 2012). The solution employed precise orbits and clocks provided by Wuhan University (Guo et al. 2016).

The weights of DD pseudoranges are derived by means of the law of variance propagation, taking into account interstation and inter-satellite correlations instigated by double differencing, as well as a priori variances of undifferenced observations (Paziewski and Wielgosz 2014). Typically, a function of satellite elevation is used for observation weighting in GNSS positioning. Then, a priori standard deviation of the pseudorange is derived adopting zenith code $\operatorname{STD}\left(\sigma_{P}\right)$ and satellite elevation $(e l)$ as follows:

$\sigma_{P_{k}^{m}}=\sqrt{\sigma_{P}^{2} / \sin ^{2}\left(e l_{k}^{m}\right)}$

Since the C/N0 is one of the key indicators assessing the quality of GNSS observations, this ration can be alternatively and efficiently employed for stochastic modeling (Hartinger and Brunner 1998; Braasch and van Dierendonck 
1999; Kuusniemi et al. 2007). With the use of high-grade receivers, both approaches (elevation and $\mathrm{C} / \mathrm{N} 0$ dependent) show a comparable performance due to a clear relationship between $\mathrm{C} / \mathrm{N} 0$ and satellite elevation angle. However, a clear benefit from $\mathrm{C} / \mathrm{N} 0$-dependent stochastic models is obtained in the signal-degraded environment (Medina et al. 2018). In this case, a priori code observation error used in the weighting process can be approximated by the following equation (Langley 1996):

$\sigma_{P_{k}^{m}}=\sqrt{\frac{\alpha B_{L}}{c / n_{0}}} \lambda_{c}$

where $c / n_{0}$ is the carrier-to-noise density, which equals $10^{\left(\mathrm{C} / \mathrm{N}_{0}\right) / 10}$ for $\mathrm{C} / \mathrm{N}_{0}$ given in $\mathrm{dB}-\mathrm{Hz}, \lambda_{c}$ corresponds to a wavelength of C/A or P-code (29.305 m or $293.05 \mathrm{~m}), B_{L}$ denotes the equivalent code loop noise bandwidth $(\mathrm{Hz})$ typically reaching up to several $\mathrm{Hz}$ and $\alpha$ denotes the dimensionless DLL (delay lock loop) discriminator correlator factor. The values of constants $B_{L}$ and $\alpha$ were adopted after Langley (1996).

As previously revealed, the smartphone observations have low $\mathrm{C} / \mathrm{N} 0$ and are less dependent on satellite signal elevation. Hence, in this case study, we present a collection of results derived with both weighting schemes.

\section{Performance of stand-alone positioning and potential improvement from code relative processing}

We show the propagation of high noise of smartphone pseudoranges into position estimates and the benefits of relative positioning. The coordinate estimates are obtained from 13-h dataset (4:00-17:00 UTC), which are split into a defined length of observation sessions of 10 and $20 \mathrm{~min}$. These solutions are compared to benchmark coordinates of high precision provided by static relative positioning.

To assess the stand-alone smartphone positioning, we process code pseudoranges in the static mode. Considering the level of measurement noise characterized previously, we cannot expect a high-precision solution. In the case of 10-min sessions, the standard deviation of coordinate residuals reaches $4.1 \mathrm{~m}, 2.7 \mathrm{~m}$ and $7.0 \mathrm{~m}$ for NEU components, respectively, with mean offset with respect to the benchmark position of $1.8 \mathrm{~m},-0.4 \mathrm{~m}$ and $3.1 \mathrm{~m}$. In this case, the study of a longer occupation time $(20 \mathrm{~min})$ did not lead to accuracy enhancement. The high variability of coordinate residuals is evident in Fig. 13 (green and blue dots), where we can notice several solutions with coordinate residuals exceeding $10-15 \mathrm{~m}$ and $20 \mathrm{~m}$ for $\mathrm{NE}$ and $\mathrm{U}$ components, respectively. A similar level of accuracy of stand-alone positioning with the smartphone was reported by Siddakatte

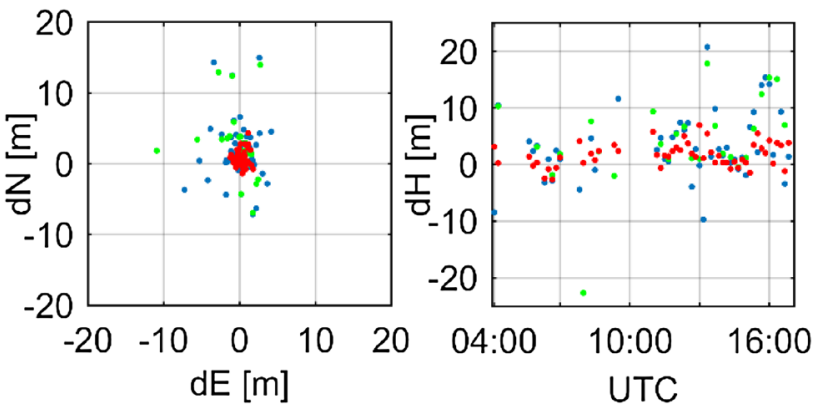

Fig. 13 Coordinate residuals obtained from stand-alone smartphone positioning of $10 \mathrm{~min}$ (blue), $20 \mathrm{~min}$ (green) sessions, and static relative positioning with $13-\mathrm{km}$-long baseline and $10 \mathrm{~min}$ session (red)

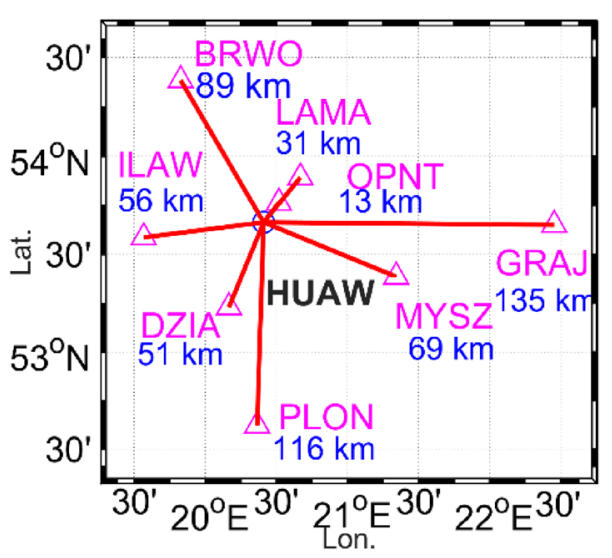

Fig. 14 Network of stations employed in relative positioning. OPNT and DZIA serve as reference stations in short and medium baseline cases, respectively, whereas LAMA, ILAW, DZIA, MYSZ serve as references in medium-range multi-station and BRWO, PLON, GRAJ in long-range multi-station solution. HUAW is the site occupied by the smartphone

et al. (2017) and Zhang et al. (2018b). Such results obviously indicate a need of positioning accuracy enhancement, which we intend to obtain with code relative positioning. Hence, static positioning was performed using a single-baseline mode with baselines of $13 \mathrm{~km}$ and $51 \mathrm{~km}$ length, as well as a multi-station mode of medium-range (31-69 km) and of long-range ( $89-135 \mathrm{~km})$ baselines. Figure 14 illustrates the location of the smartphone with respect to the reference stations and baselines. To verify the hypothesis of high impact of $\mathrm{C} / \mathrm{N} 0$ on coordinate estimates, we have employed two aforementioned weighting schemes: satellite elevation and C/NO dependent.

Table 2 summarizes the positioning accuracy and provides empirical standard deviation of the coordinate residuals. In these cases, the STDs of the coordinate residuals were in the range of $0.8-1.5 \mathrm{~m}, 0.6-1.4 \mathrm{~m}$ and $1.6-2.8 \mathrm{~m}$ for NEU components, respectively, with corresponding mean offset to benchmark position equal to $0.7-0.8 \mathrm{~m}$, about $0.2 \mathrm{~m}$, 
Table 2 Statistics of codebased relative positioning in the function of baseline range and duration of observing session. STD denotes coordinate residuals standard deviation obtained with respect to benchmark position

\begin{tabular}{|c|c|c|c|c|c|c|c|c|}
\hline \multirow[b]{2}{*}{ 10-min } & \multicolumn{2}{|c|}{$\begin{array}{l}\text { Short single base- } \\
\text { line }(13 \mathrm{~km})\end{array}$} & \multicolumn{2}{|c|}{$\begin{array}{l}\text { Medium single } \\
\text { baseline }(51 \mathrm{~km})\end{array}$} & \multicolumn{2}{|c|}{$\begin{array}{l}\text { Multi-station } \\
\text { medium range } \\
(31-69 \mathrm{~km})\end{array}$} & \multicolumn{2}{|c|}{$\begin{array}{l}\text { Multi-station } \\
\text { long range } \\
(89-135 \mathrm{~km})\end{array}$} \\
\hline & & & & & & & & \\
\hline Weighting scheme & el. & C/NO & el. & C/N0 & el. & C/NO & el. & $\mathrm{C} / \mathrm{NO}$ \\
\hline $\operatorname{STD}_{N}(\mathrm{~m})$ & 1.47 & 1.09 & 1.48 & 1.15 & 1.48 & 1.09 & 1.50 & 1.15 \\
\hline $\operatorname{STD}_{\mathrm{E}}(\mathrm{m})$ & 0.84 & 0.69 & 0.84 & 0.69 & 0.83 & 0.68 & 1.37 & 0.94 \\
\hline $\operatorname{STD}_{U}(\mathrm{~m})$ & 2.80 & 2.11 & 2.81 & 2.17 & 2.79 & 2.15 & 2.74 & 2.22 \\
\hline \multicolumn{9}{|l|}{ 20-min } \\
\hline $\mathrm{STD}_{\mathrm{N}}(\mathrm{m})$ & 1.14 & 0.81 & 1.19 & 0.84 & 1.21 & 0.87 & 1.29 & 1.18 \\
\hline $\operatorname{STD}_{\mathrm{E}}(\mathrm{m})$ & 0.68 & 0.60 & 0.68 & 0.60 & 0.67 & 0.61 & 0.64 & 0.65 \\
\hline $\operatorname{STD}_{U}(\mathrm{~m})$ & 2.34 & 1.67 & 2.39 & 1.62 & 2.39 & 1.85 & 2.35 & 2.21 \\
\hline
\end{tabular}

and 1.7-2.0 $\mathrm{m}$ depending on the processing strategy. Comparing the performance of the relative positioning to the stand-alone one, we notice a clear benefit from the doubledifferenced observation processing in terms of both position repeatability and mean offset. Relative mode reduced the dispersion of position about 3-4 times with respect to the stand-alone solution.

Table 2 also shows that the application of C/N0-dependent weighting scheme significantly reduces the coordinate STD with respect to the elevation-dependent weighting function. For instance, in the case of the 10-min session, we obtained the enhancement of coordinate STD fitting the range of $28-35 \%, 21-45 \%, 23-32 \%$, for the NEU components, respectively. Specifically, the coordinate STD for the short single-baseline solution reduces from $1.47 \mathrm{~m}$ for $\mathrm{N}$, $0.84 \mathrm{~m}$ for $\mathrm{E}$ and $2.80 \mathrm{~m}$ for $\mathrm{U}$ component, to $1.09 \mathrm{~m}, 0.69 \mathrm{~m}$ and $2.11 \mathrm{~m}$, respectively. Hence, we can conclude that the commonly applied elevation weighting scheme is not optimal for smartphone-based positioning due to the lower dependency of the carrier-to-noise density ratio on satellite elevation. Thus, in such a case, this should be replaced by C/N0-dependent scheme.

Interesting lessons can be learned from the comparison of results obtained with different baselines length. We may draw the conclusion that the impact of baseline length is of lower importance, and thus, tropospheric and ionospheric refraction is not dominating the error budget for relative positioning with a range up to $100 \mathrm{~km}$. We obtain relatively similar values of coordinate STD for single baselines of 13 and $51 \mathrm{~km}$ and for a multi-station solution of medium-range baselines. However, a slightly lower precision was obtained for long baselines. With 20-min sessions, baselines up to dozens of kilometers and the $\mathrm{C} / \mathrm{N} 0$-dependent weighting scheme, it is feasible to achieve a smartphone position with STDs of $0.8 \mathrm{~m}, 0.6 \mathrm{~m}$ and $1.6 \mathrm{~m}$, for NEU components, respectively. The results indicate that a significant enhancement of the smartphone positioning can be obtained employing a relatively sparse network of reference stations with baselines up to $135 \mathrm{~km}$. Hence, long-range pseudorange solution may reach a similar level of accuracy as phase plus pseudorange stand-alone positioning of the smartphone with disabled duty cycling as presented in Zhang et al. (2018b).

\section{Conclusions and future work}

We assessed both the measurement quality and the potential benefit of relative positioning to improve the positioning accuracy of smart devices. The study shows that smartphone observables suffer not only from high measurement noise combined with multipath effect and low signal-to-noise ratio but also from several anomalies driven by the duty-cycling mode. The results show increasing divergence between phase and code data after the activation of the duty cycling, which points toward an inconsistency between smartphone phase and code clocks. Moreover, activation of the duty cycling caused repeated variations in code data, which is also considered a receiver clock-related effect. Finally, double-differenced phase observables exposed unwanted drift during the activated duty-cycling mode. In contrary to previously detected effects, this long-term change does not reveal a coincidence between different pairs of satellites. Such effect efficiently prevents integer ambiguity resolution and hence is a severe constraint limiting the application to high-precision positioning techniques such as RTK or PPP. Based on the analysis of the observables, we may expect to address the requirements of precise positioning, providing observables derived from a smartphone without the duty cycle.

To improve the performance of smartphone positioning, we employed code relative positioning of different range, taking advantage of the national permanent GNSS network. We conclude that in such a case the dominating factor, degrading the performance of positioning, is the quality of observations. Even for baselines of over $100 \mathrm{~km}$, it is considered as the main constraint, rendering other sources of 
errors to be less important. The results show that long-range relative mode might significantly enhance smartphone positioning with respect to the stand-alone solution and provide the submeter level of horizontal coordinate accuracy. This is feasible taking advantage of $\mathrm{C} / \mathrm{N} 0$-dependent weighting scheme which was superior to the satellite elevation one.

We should note that these statements hold true only for the devices with low-quality phase observations being subject of enabled duty cycling, the gradual accumulation of errors, or the presence of arbitrary starting phase offset. Hence, our future work will involve not only the application of chipsets with inactive duty cycling but also the most recent chipsets such as BCM47755 by Broadcom capable of tracking dual-frequency data, creating an opportunity for the handling of ionospheric delay.

Acknowledgements The work in this contribution was supported by the National Science Centre, Poland: Project No. 2016/23/D/ ST10/01546.

Open Access This article is distributed under the terms of the Creative Commons Attribution 4.0 International License (http://creativeco mmons.org/licenses/by/4.0/), which permits unrestricted use, distribution, and reproduction in any medium, provided you give appropriate credit to the original author(s) and the source, provide a link to the Creative Commons license, and indicate if changes were made.

\section{References}

Amiri-Simkooei AR, Tiberius CCJM (2007) Assessing receiver noise using GPS short baseline time series. GPS Solut 11(1):21-35

Boehm J, Niell A, Tregoning P, Schuh H (2006) Global Mapping Function (GMF): A new empirical mapping function based on numerical weather model data. Geophys Res Lett 33(7):L07304

Bona P (2000) Precision, cross correlation, and time correlation of GPS phase and code observations. GPS Solut 4(2):3-13

Braasch MS, van Dierendonck AJ (1999) GPS receiver architectures and measurements. In: Proceedings of the IEEE, vol 87(1), pp 48-64. https://doi.org/10.1109/5.736341

de Bakker PF, Tiberius CCJM, van der Marel H, van Bree RJP (2012) Short and zero baseline analysis of GPS L1 C/A, L5Q, GIOVE E1B, and E5aQ signals. GPS Solut 16(1):53-64

Engelbrecht J, Booysen MJ, van Rooyen GJ, Bruwer FJ (2015) Survey of smartphone-based sensing in vehicles for intelligent transportation system applications. IET Intell Transp Syst 9(10):924-935

Gill M, Bisnath S, Aggrey J, Seepersad G (2017) Precise Point Positioning (PPP) using Low-Cost and Ultra-Low-Cost GNSS Receivers. In: Proceedings of the ION GNSS + 2017, Institute of Navigation, Portland, Oregon, USA, September 25-29, pp 226-236

Guo J, Xu X, Zhao Q, Liu J (2016) Precise orbit determination for quad-constellation satellites at Wuhan University: strategy, result validation, and comparison. J Geodesy 90(2):143-159

Håkansson M (2019) Characterization of GNSS observations from a Nexus 9 Android tablet. GPS Solut 23:21. https://doi.org/10.1007/ s10291-018-0818-7

Hartinger H, Brunner FK (1998) Attainable accuracy of GPS measurements in engineering surveying. In: Proceedings of FIG XXI international conference, Brighton, UK, July 21-25, pp 18-31
Hauschild A, Montenbruck O, Sleewaegen JM, Huisman L, Teunissen PJG (2012) Characterization of compass M-1 signals. GPS Solutions 16(1):117-126

Humphreys TE, Murrian M van Diggelen F, Podshivalov S, Pesyna KM (2016) On the Feasibility of cm-Accurate Positioning via a Smartphone's Antenna and GNSS Chip, 6 PROC. IEEE/ION PLANS 2016 Savannah, Georgia, USA, April 11-14, pp 232-242

Kirkko-Jaakkola M, Söderholm S, Honkala S, Koivula H, Nyberg S, Kuusniemi H (2015) Low-Cost Precise Positioning Using a National GNSS Network. In: Proceedings of the ION GNSS + 2015, Institute of Navigation, Tampa, Florida, USA, September 14-18, pp 2570-2577

Kuusniemi H, Wieser A, Lachapelle G, Takala J (2007) User-level reliability monitoring in urban personal satellite-navigation. IEEE Trans Aerosp Electron Syst 43(4):1305-1318

Langley RB (1996) GPS receivers and the observables. In: Kleusberg A, Teunissen PJG (eds) GPS for geodesy. Lecture notes in earth sciences, vol 60. Springer, Berlin

Laurichesse D, Rouch C, Marmet FX, Pascaud M (2017) Smartphone applications for precise point positioning. In: Proceedings of the ION GNSS + 2017, Institute of Navigation, Portland, Oregon, USA, September 25-29, pp. 171-187

Liu Q, Ying R, Wang Y, Qian J, Liu P (2018) Pseudorange double difference algorithm based on duty-cycled carrier phase smoothing on low-power smart devices. In: Sun J, Yang C, Guo S (eds) China satellite navigation conference (CSNC) 2018 Proceedings. CSNC 2018. Lecture Notes in Electrical Engineering, vol 497. Springer, Singapore

Liu W, Shi X, Zhu F, Tao X, Wang F (2019) Quality analysis of multi-GNSS raw observations and a velocity-aided positioning approach based on smartphones. Adv Space Res 63(8):2358-2377

Medina D, Gibson K, Ziebold R, Closas P (2018) Determination of pseudorange error models and multipath characterization under signal-degraded scenarios. In: Proceedings of the ION GNSS +, Institute of Navigation, Miami, Florida, USA, September 24-28

Odolinski R, Teunissen PJG (2017) Low-cost, 4-system, precise GNSS positioning: a GPS, Galileo, BDS and QZSS ionosphere-weighted RTK analysis. Meas Sci Technol 28(12):125801

Paziewski J (2016) Study on desirable ionospheric corrections accuracy for network-RTK positioning and its impact on time-to-fix and probability of successful single-epoch ambiguity resolution. Adv Space Res 57(4):1098-1111

Paziewski J, Wielgosz P (2014) Assessment of GPS + Galileo and multi-frequency Galileo single-epoch precise positioning with network corrections. GPS Solut 18(4):571-579

Paziewski J, Sieradzki R, Baryla R (2018) Multi-GNSS high-rate RTK, PPP and novel direct phase observation processing method: application to precise dynamic displacements detection. Meas Sci Technol 29:035002

Pesyna KMJ, Heath RWJ, Humphreys TE (2014) Centimeter positioning with a smartphone-quality GNSS antenna. In: Proceedings of the ION GNSS 2014, Institute of Navigation, Tampa, Florida, USA, September 8-12, pp 1568-1577

Pirazzi G, Mazzoni A, Biagi L, Crespi M (2017) Preliminary performance analysis with a GPS + Galileo enabled chipset embedded in a smartphone. In: Proceedings of the ION GNSS + 2017 Institute of Navigation, Portland, Oregon, USA, September 25-29, pp 101-115

Riley S, Lentz W, Clare A (2017) On the path to precision - observations with android GNSS observables. In: Proceedings of the ION GNSS + 2017, Institute of Navigation Portland, Oregon, USA, September 25-29, pp 116-129 
Saeedi S, Moussa A, El-Sheimy N (2014) Context-Aware Personal Navigation Using Embedded Sensor Fusion in Smartphones. Sensors 14(4):5742-5767

Siddakatte R, Broumandan A, Lachapelle G (2017) Performance evaluation of smartphone GNSS measurements with different antenna configurations. In: Proceedings of the royal institute of navigation international navigation conference, 27-30 November 2017, Brighton

Specht C, Dabrowski PS, Pawelski J, Specht M, Szot T (2019) Comparative analysis of positioning accuracy of GNSS receivers of Samsung Galaxy smartphones in marine dynamic measurements. Adv Space Res 63(9):3018-3028

Takasu T, Yasuda A (2008) Evaluation of RTK-GPS performance with low-cost single-frequency GPS receivers. In: Proceeding of the international symposium on GPS/GNSS 2008 Tokyo, pp 852-861

Wang D, Park S, Fesenmaier DR (2011) The Role of Smartphones in Mediating the Touristic Experience. Journal of Travel Research 51(4):371-387

Wang L, Groves PD, Ziebart MK (2012) Multi-constellation GNSS performance evaluation for urban canyons using large virtual reality city models. J Navig 65(03):459-476

Wielgosz P, Paziewski J, Krankowski A, Kroszczyński K, Figurski M (2012) Results of the application of tropospheric corrections from different troposphere models for precise GPS rapid static positioning. Acta Geophys 60(4):1236-1257

Wisniewski B, Bruniecki K, Moszynski M (2013) Evaluation of RTKLIB's positioning accuracy using low-cost GNSS receiver and ASG-EUPOS. Int J Mar Navig Saf Sea Transp 7(1):79-85

Zhang K, Jiao F, Li J (2018a) The Assessment of GNSS Measurements from Android Smartphones. In: Sun J, Yang C, Guo S (eds) China Satellite Navigation Conference (CSNC) 2018 Proceedings. CSNC 2018. Lecture Notes in Electrical Engineering, vol 499. Springer, Singapore

Zhang X, Tao X, Zhu F, Shi X, Wang F (2018b) Quality assessment of GNSS observations from an Android N smartphone and positioning performance analysis using time differenced filtering approach. GPS Solut 22:70. https://doi.org/10.1007/s1029 1-018-0736-8

Realini E, Caldera S, Pertusini L, Sampietro D (2017) Precise GNSS positioning using smart devices. Sensors 17(10):2434. https://doi. org/10.3390/s 17102434

Publisher's Note Springer Nature remains neutral with regard to jurisdictional claims in published maps and institutional affiliations.

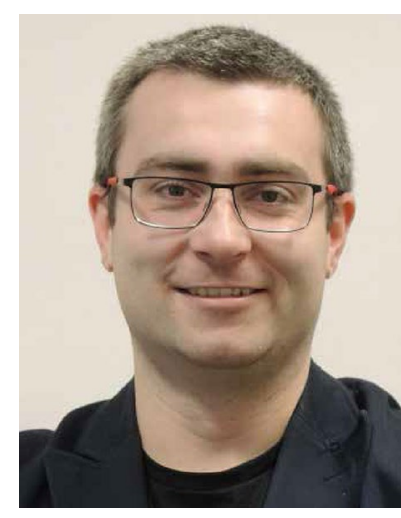

Jacek Paziewski is an Associate Professor at the University of Warmia and Mazury in Olsztyn. His research interests cover the integration of multi-constellation observations in relative and precise point positioning, modeling of the ionospheric delays and high-rate GNSS data processing.

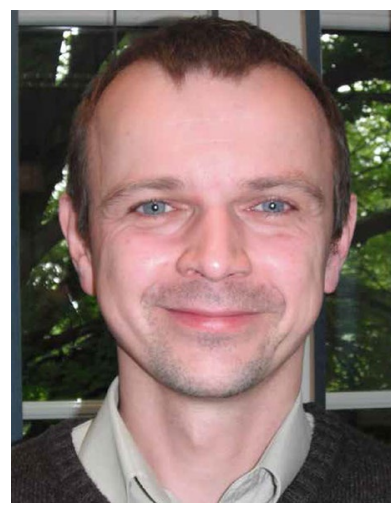

Rafal Sieradzki is an Assistant Professor at the University of Warmia and Mazury (UWM) in Olsztyn. His research interest focuses on GNSS-based monitoring of ionospheric disturbances at high latitudes. He is also engaged in the development of algorithms aimed at mitigation of ionospheric delays in GNSS positioning.

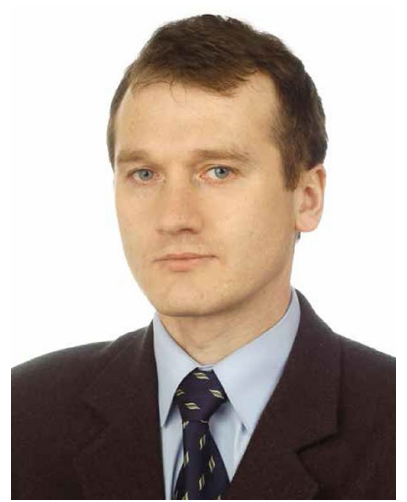

Radoslaw Baryla is an Assistan Professor at the University of Warmia and Mazury in Olsztyn. His main research interests include the application of GNSS to surveying engineering, precise leveling as well as deformation monitoring. 